\title{
Responsiveness to volume change of calcinosis cutis utilizing dual-energy computed tomography in a patient with diffuse cutaneous systemic sclerosis treated with alendronate
}

Background: A 33-year-old pleasant Emirati lady presented to Cleveland Clinic Abu Dhabi with an established diagnosis of diffuse cutaneous systemic sclerosis and calcinosis cutis of 7 years duration from the first non-Raynaud's disease manifestation. She was experiencing pain, open wounds and visible palpable stones involving the knees. Despite treatment over the years with different medications including oral steroids, colchicine, sodium thiosulphate infusions and mycophenolate mofetil, her illness progressed. She was started on alendronate 70 mg weekly on March of 2017 as a treatment option for refractory calcinosis cutis.

We examined the usefulness of dual-energy computed tomography (DECT) in excluding monosodium urate (MSU) crystal deposition and further quantified the amount of calcific deposits to assess responsiveness to alendronate after being on the medication for 1 year. There was a clinically meaningful improvement in knee pain, wound healing and volume of calcium deposits pre and post treatment with alendronate. DECT scan is a useful tool that can further characterize the morphology, density and distribution of the calcinosis within the soft tissues as well as providing the added benefit of volume quantification to assess responsiveness to treatments.

Keywords: calcinosis • systemic sclerosis • dual-energy computed tomography

\section{Introduction}

A 33-year-old pleasant Emirati lady presented to the Rheumatology Clinic at Cleveland Clinic Abu Dhabi with an established diagnosis of diffuse cutaneous systemic sclerosis $(\mathrm{dcSS} c)$ of 7 years duration from the first non-Raynaud's disease manifestation. Since the time of diagnosis she was experiencing progressively worsening painful ulcers associated with soft tissue swelling and recurrent infections involving the knees and was diagnosed with calcinosis cutis. Despite treatment over the years with different disease modifying drugs including oral steroids, colchicine, sodium thiosulphate infusions and mycophenolate mofetil her illness progressed. Physical exam of the knees revealed an erythematous open wound involving the anterior aspect of the knees with visible palpable white stones. Alendronate 70 mg weekly was started on March 2017 and dualenergy computed tomography was utilized to assess the responsiveness to treatment after being on the medication for 1 year.
Calcinosis cutis is defined as the deposition of insoluble calcium salt in the skin and subcutaneous soft tissues, which is frequently manifested in patients with systemic sclerosis (Ssc) $[1,2]$. It occurs in about $25 \%$ of patients with SSc and can occur in any subset of SSc but is often seen in limited cutaneous systemic sclerosis [3]. Calcium hydroxyapatite is reported to be the major constituent of SSc-associated calcinosis [4]. Deposits are commonly found in pressures areas of the elbows, knees, and hands [5]. Patients with calcinosis tend to be older with longer disease duration from initial non-RP symptom [6]. Calcium deposits can be palpable on exam and diagnosed by plain radiography [7]. Additional types of imaging that can evaluate calcinosis including ultrasound [8], and dualenergy computed tomography $[9,10]$.

We examined the usefulness of dual-energy computed tomography (DECT) in-

- exclusion of monosodium urate (MSU) crystal deposition and

\section{Rajaie Namas ${ }^{* 1}$ \& Esat Memisoglu'}

'Department of Internal Medicine, Division of Rheumatology, Cleveland Clinic Abu Dhabi, Abu Dhabi, UAE

${ }^{2}$ Department of Radiology, Division of Musculoskeletal Imaging, Cleveland Clinic Abu Dhabi, Abu Dhabi, UAE

*Author for correspondence: namasr@clevelandclinicabudhabi.ae 
- quantification of the amount of calcific deposits as this can guide in the assessment of treatment response in a patient with symptomatic SSc-associated calcinosis.

We quantified the amount of calcific deposits prior to starting alendronate $70 \mathrm{mg}$ weekly and a year later to assess treatment response. There was clinically meaningful improvement in pain, and wound healing (images of the wounds not submitted). In addition, there was a meaningful decline in the pre and post treatment volume of calcific deposits in both knees, more in the left knee. Figures 1-2 (Right knee pre: $21.74 \mathrm{~cm}^{3}$; post: $18.03 \mathrm{~cm}^{3}$ ), Left knee (pre: $23.99 \mathrm{~cm}^{3}$; post: $\left.17.13 \mathrm{~cm}^{3}\right)$.

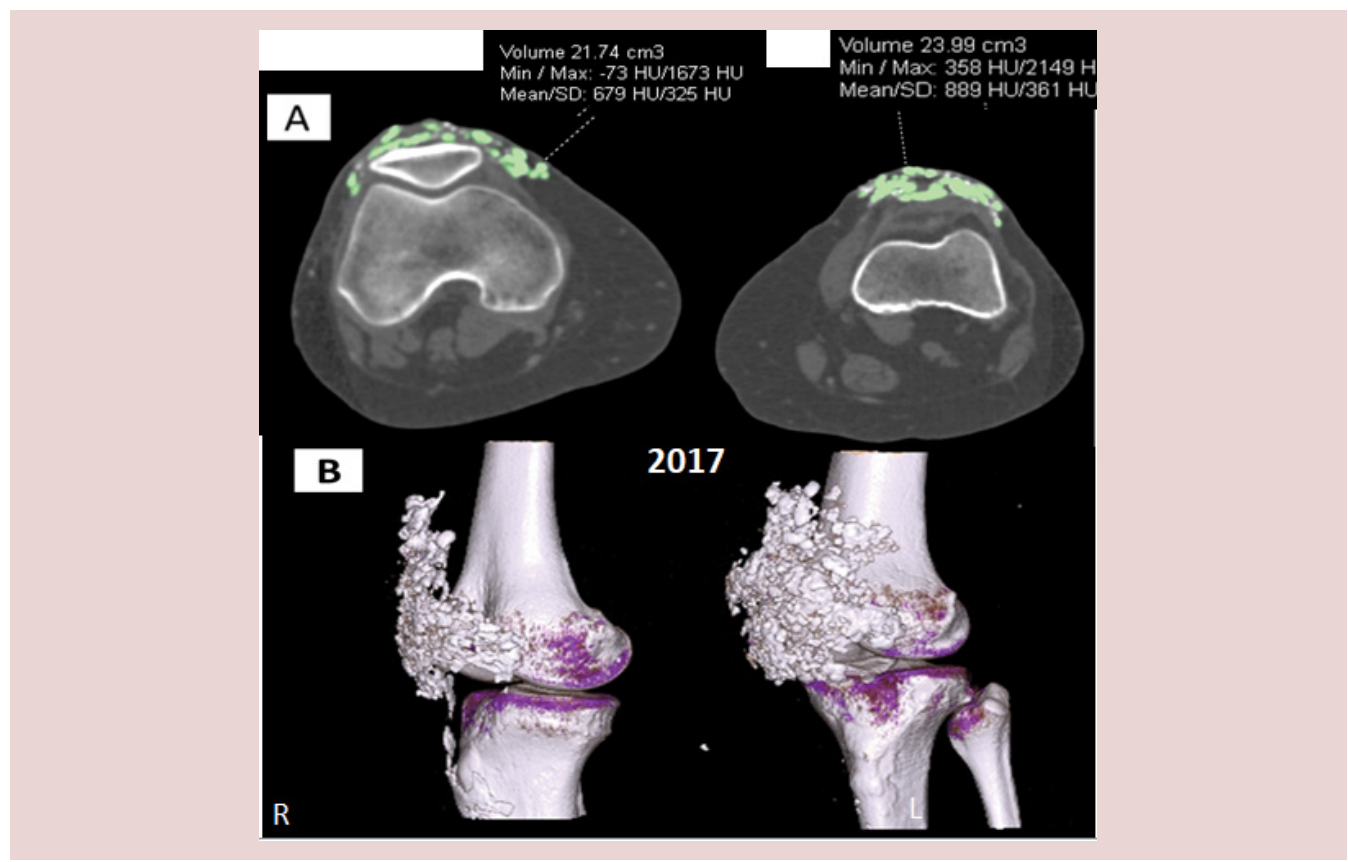

Figure 1. Dual energy computed tomography with color rendering obtained in 2017 revealing extensive soft tissue calcium deposits in both anterior knees; (A) axial tomographic and (B) 3-D volumetric images

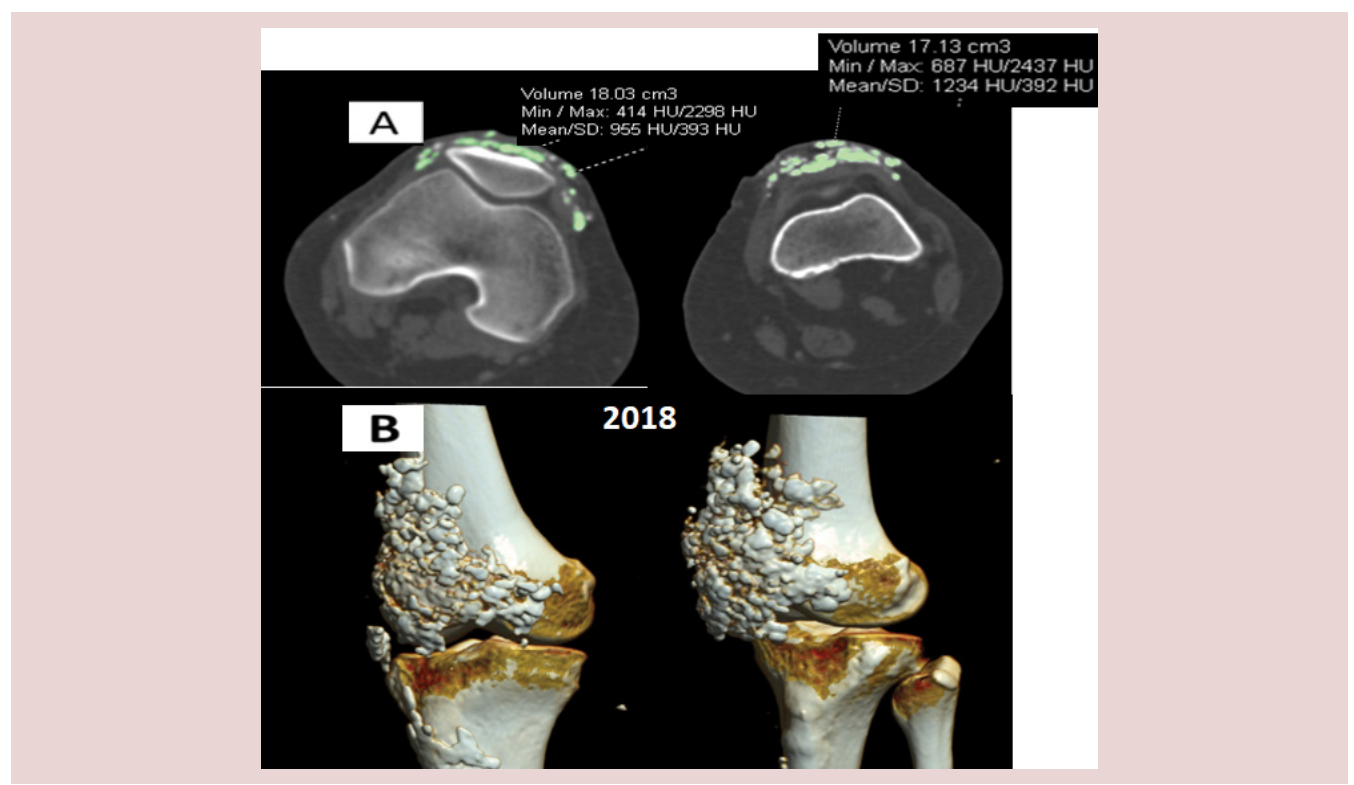

Figure 2. Dual energy computed tomography with color rendering obtained in 2018 revealing reduction of extensive soft tissue calcium deposits in both anterior knees; $(A)$ axial tomographic and (B) 3-D volumetric images 
DECT scan can further characterize the morphology, density and distribution of the calcinosis within the soft tissues as well as providing the added benefit of volume quantification.

CT imaging is an option to be considered and utilized in the management of SSc -associated calcinosis which can give more insight in locating these deposits within the soft tissues and can guide in assessing responsiveness to treatments in refractory cases of calcinosis.

\section{Acknowledgement}

I want to thank the patient that give us all the trust to treat her medical condition.

\section{References}

1. Young A, Namas R, Dodge C et al. Hand Impairment in Systemic Sclerosis: Various Manifestations and Currently Available Treatment. Curr. Treatm. Opt. Rheumatol. 2, 252-269 (2016)

2. Valenzuela A, Chung L. Calcinosis: pathophysiology and management. Curr. Opin. Rheumatol. 27, 542-8 (2015).
3. Morrisroe KB, Nikpour M, Proudman SM. Musculoskeletal Manifestations of Systemic Sclerosis. Rheumatic Diseases Clinics of North America. 41(3), 507-18 (2015).

4. Gutierrez A, Wetter DA. Calcinosis cutis in autoimmune connective tissue diseases. Dermatol. Ther. 25, 195-206 (2012).

5. Hsu V, Bramwit M, Schlesinger N. Use of dual-energy computed tomography for the evaluation of calcinosis in patients with systemic sclerosis. Clin. Rheumatol. 34, 1557-61 (2015).

6. Chander S, Gordon P. Soft tissue and subcutaneous calcification in connective tissue diseases. Curr. Opin. Rheumatol. 24(2), 158-64 (2012).

7. Chung L, Valenzuela A, Fiorentino D et al. Validation of a novel radiographic scoring system for calcinosis affecting the hands of patients with systemic sclerosis. Arthritis. Care. Res. 67(3), 425-430 (2015).

8. Elhai $\mathrm{M}$, Guerini $\mathrm{H}$, Bazeli $\mathrm{R}$ et al. Ultrasonographic hand features in systemic sclerosis and correlates with clinical, biologic, and radiographic findings. Arthritis. Care. Res. 64(8), 1244-1249 (2012).

9. Hsu V, Bramwit M, Schlesinger N. Dual-energy computed tomography for the evaluation of calcinosis in systemic sclerosis. J. Rheumatol. 42, 345-6 (2015).

10. Freire V, Becce F, Feydy A et al. MDCT imaging of calcinosis in systemic sclerosis. Clin. Radiol. 68(3), 302 309 (2013). 